\title{
LE MOSCHE BIANCHE. \\ PERCHÉ I GIOVANI ATTIVISTI DI PARTITO \\ SONO POCHI?
}

di Ettore Recchi

Giovani e partiti: una crisi senza fine

I partiti politici detengono il triste primato di essere le istituzioni pubbliche in cui $i$ giovani italiani nutrono minor fiducia - meno che nella burocrazia statale, nel governo, nella magistratura, nelle forze di polizia, nell'esercito, nella Chiesa, nei sindacati, nelle banche, nei giornali e nelle televisioni (Buzzi et al. $1997,382)^{1}$. Per quanto aggravatosi in tempi recenti, questo discredito ha radici lontane e trova espressione in un distacco crescente, dagli anni settanta in poi, dei giovani dalla vita dei partiti. Generazione dopo generazione, stando alle survey condotte negli ultimi cinque lustri, i cittadini dai 18 ai 25 anni che prendono parte regolarmente alle attività di qualche organizzazione politica sono passati dal $7,3 \%$ del 1970 , al $6,2 \%$ del 1983, al 4,1\% del 1987, al 2,7\% del 1992 e del 1996 (Tullio-Altan e Marradi 1976, 472; Cavalli e de Lillo 1993, 286; Buzzi et al. 1997, 419). Più precisamente, gli individui tra i 20 e i 24 anni che negli ultimi sei mesi hanno svolto «attività gratuita per un partito» ammontano nel 1994 ad un misero $1,8 \%$ - meno di un terzo della quota di attivisti di partito che si ritrova nella popolazione fra i 35 e i 44 anni d'età (Istat 1996, 143). Dati di

Versioni precedenti di questo articolo sono state presentate al seminario «Valori politici e muove generacioni nell Europe contemporaneas onganizato dal Centro Interuniversitario di Sociologia Politice presso la Facoltì di Scienze Politicbe aC. Alfierio di Firenze il 25-26 settembre 1997 e ad un incontro promosso dal Groupe dEtude et de Recherche sur les Modalités du Militantisme dell Association Frangaise de Science Politique presso IInstitut dE Etudes Politiques di Parigi il 12 febbraio 1998.

1 Tra i giovani di 15-29 anni, nel $19 \%$ solo P'1,3\% ripone umoltan e il 12,0\% vabbastanzao fiducia nei partiti. Purtroppo questo item non era stato incluso nelle precedenti rilevarioni periodiche dello Iard. 
fonte partitica, del resto, confermano il quadro: a cavallo fra il 1995 e il 1996 le organizzazioni giovanili dei partiti contavano 204.000 membri (Turi 1997, 126) - ossia, il 2\% della popolazione tra i 19 e 29 anni (Istat 1995, 102)2.

Per cercare di comprendere meglio perché i giovani siano così refrattari a qualsiasi coinvolgimento partitico (persino nella forma minima del tesseramento), sembra utile mettere a fuoco i casi devianti - quei pochissimi esponenti delle nuove generazioni, cioè, che invece alla vita di partito dedicano tempo ed energie. Dall'individuazione dei caratteri distintivi di queste «mosche bianche» è forse possibile ricavare, per contrasto, alcune indicazioni sui fattori che trattengono la quasi totalità dei loro coetanei dal far politica.

Nella prima parte dell'articolo presenterò alcuni modelli di spiegazione delle condizioni causali che soggiacciono all'attivismo di partito in giovane età. Nella sezione successiva questi modelli saranno sottoposti a controllo empirico a partire da dati di confronto tra $\mathrm{i}$ giovani attivisti di partito e i loro coetanei apolitici. Le implicazioni dei risultati ottenuti saranno infine discusse alla luce anche di precedenti analisi delle carriere politiche di parlamentari ed ex militanti delle organizzazioni giovanili dei principali partiti italiani.

Perché in politica? Sulle radici del coinvolgimento politico dei giovani attivisti

Ipotesi circa le condizioni che inducono alcuni individui anziché altri ad attivarsi politicamente sono state formulate in diverse aree di ricerca - la partecipazione politica, il reclutamento politico, la socializzazione delle élites - dando vita ad alcuni modelli interpretativi che percorrono, in elaborazioni più o meno esplicite, la letteratura. Ad essi se ne aggiungerà - nella sintetica rassegna che segue - un altro, cui si è dato il nome di modello di identificazione razionale.

Il modello di compensazione. Per gli psicologi politici di matrice freudiana le radici della personalità politica sono da ricer-

2 Beninteso, questa quota ha solamente valore di stima in quanto, da un lato, i limiti di età delle organizzazioni giovanili di partito variano tra i 14 e i 31 anni e, d'altra parte, in taluni casi ai giovani iscritti è consentito avere la tessera del partito senza una registrazione nelle fila dell'organizzazione giovanile. 
carsi in un deficit affettivo subito durante l'infanzia in ambito familiare e poi amplificatosi in un'adolescenza particolarmente solitaria e infelice. Lo schema analitico di riferimento è stato sviluppato originariamente da Lasswell, secondo il quale «il vero politico impara ad usare il mondo degli oggetti pubblici come strumento per alleviare le sofferenze che caratterizzano il suo ambiente intimo. [Per il politico] i desideri di deferenza, frustrati o viziati nella cerchia degli affetti, trovano espressione nell'ambiente secondario» (Lasswell 1950, 17). E soprattutto alla repressione ad opera della figura paterna che, in questa cornice interpretativa, si imputa la formazione di una bassa stima di sé destinata a segnare la personalità del politico e ad alimentarne il desiderio di riscatto tramite la conquista di influenza e consenso. Benché si ritenga che la vera e propria attivazione politica abbia luogo come esito di dinamiche psicologiche successive (specialmente utili per spostare su un oggetto collettivo il proprio risentimento inconscio, secondo la nota formula lasswelliana di sviluppo della personalità del politico) $)^{3}$, l'esigenza di compensare un'immagine di sé poco soddisfacente, sperimentata in maniera particolarmente sofferta durante l'adolescenza in forma di senso di impotenza e di separazione dal gruppo dei pari, ne costituisce la precondizione necessaria ${ }^{4}$.

«L'ipotesi chiave» - nella visione di Lasswell $(1948,39)$ - è che «la persona che cerca di conquistare il potere ne è attratto in quanto strumento di compensazione della propria deprivazione» - deprivazione, in particolare, di affetto, amicizia e legami di gruppo negli anni dell'adolescenza. Più precisamente, «ci si aspetta che il potere consenta di superare una bassa stima di sé sollecitando un cambiamento o dei tratti del sé o dell'ambiente in cui esso funziona» (ibidem). In termini operativi, vi è dunque $\mathrm{da}$ credere che l'impegno politico aumenti l'autostima dell'attivista attraverso una modifica del suo senso soggettivo di con-

3 La formula è: $\langle p| d\} r=P$, dove $p$ equivale a motivi privati; $d$ equivale a spostamento (displacement) su un oggetto pubblico; $r$ equivale a razionalizzazione in termini di interesse pubblico; $P$ equivale a uomo politico; e $\}$ equivale a "trasformato in"» (Lasswell 1977, 75-76).

+ Una variante di questo modello, detta «modello di attualizzazione» (Carlson e Hyde 1980), deriva dall'ipotesi, avanzata in primo luogo da Barber (1965), che anche una stima di sé straordinariamente alta costituisce una molla per l'attivazione politica in quanto porta a sottovalutare le possibilità di insuccesso nel gioco politico. In un certo senso, questa teoria assume che una forte autostima faccia apparire la politica un'impresa più agevole di quel che sembri alla maggior parte degli individui. 
trollo sulla realtà, o attraverso l'inserimento in un ambiente socialmente integrato, o fors'anche in entrambi i modi.

Il modello di compensazione non manca di tentativi di controllo empirico, che, tramite vari indicatori e tecniche, hanno cercato di rilevare il livello di attrazione per il potere degli attivisti politici (per una rassegna, cfr. Milbrath e Goel 1977, 7980). In una maniera o nell'altra, queste ricerche hanno finito col condividere la conclusione di Browning e Jacob $(1964,89)$ per cui «il mero fatto di essere un politico non implica un particolare interesse per il potere». Ciò, tuttavia, non è forse sufficiente a falsificare il modello, se non altro perché nell'ottica di Lasswell il «bisogno di potere» sembra essere un prodotto piuttosto che la radice originaria della personalità politica, che deve invece ricercarsi in una bassa stima di sé e nell'impulso a superare siffatta condiziones. Quindi, benché presa in considerazione quasi solamente in ricerche idiografiche (per eccezioni, cfr. Barber 1965; Iremonger 1970; Clarke e Donovan 1980; Carlson e Hyde 1980), l'ipotesi che carenze nel senso di controllo personale (Rotter 1966) e nel senso di socialità siano condizioni necessarie (sebbene non sufficienti) per l'attivazione politica individuale sembra rappresentare il modo più fedele di operativizzare i fattori causali ritenuti cruciali nel modello di compensazione.

Il modello di cristallizzazione. La concezione dell'attivista politico come true believer, diffusa ancor prima che in letteratura nel senso comune, trova una sua cornice teorica nel modello di cristallizzazione - come lo hanno battezzato Merelman e King (1986). Anche in questo modello interpretativo la famiglia gioca un ruolo centrale nello stimolare l'ingresso in politica, qualora a caratterizzarla siano ideali trasmessi ai figli con particolare convinzione (inculcati, per meglio dire), fondamentalmente perché «l'apprendimento nell'età pre-adulta produce negli attivisti certi abiti mentali che perdurano senza cambiamenti per tutto il corso della vita» (ibidem, 475). In conseguenza di

5 Convince meno l'obiezione secondo cui il modello sarebbe normalmente sottoposto a controllo su popolazioni inadeguate, e cioè attivisti politici in senso «istituzionale» anziché «funzionale»: «la popolazione cui si riferisce Lasswell quando parla di uomini politici non è comparabile, per dire, alla popolazione dei delegati a una convention per la nomina del candidato di un partito alla presidenza americana» (Greenstein 1977, xiv-xv). Ma questa critica non scioglie il nodo dei criteri da adottare per individuare politici in senso «funzionale» senza incorrere nella circolarità (i politici sono quelle persone che soddisfano i requisiti della teoria). 
ciò, i futuri attivisti politici maturerebbero un più saldo attaccamento a determinate configurazioni valoriali. L'attivista viene quindi concepito come un individuo «ipersocializzato» cui «risulta difficile accettare la non-realizzazione dei valori» appresi (Tapper 1976, 141-142). Ai suoi occhi, l'attivismo si configura come una risposta alla disillusione di esperire che la società non funziona secondo i canoni di riferimento dei principali agenti di socializzazione. La politica rappresenta, in altre parole, una strada da percorrere per adattare il mondo ai propri princìi. Se poi l'individuo ritrova questi stessi principi alla base dell'ideologia di un partito, la sua vocazione politica troverà in quel partito il proprio contesto di manifestazione.

In termini operativi, il modello prevede che «i giovani attivisti mostreranno di avere una somiglianza insolitamente forte con gli atteggiamenti politici degli agenti di socializzazione del loro ambiente, [nonché] atteggiamenti politici insolitamente ben strutturati per quanto è possibile rilevare con un indice di ideologizzazione» (Merelman e King 1986, 479). Inoltre, si può presumere che questi fattori - la consonanza politica con i familiari e la coerenza ideologica - esercitino un'influenza causale sull'attivismo giovanile tanto più incisiva nella misura in cui interagiscono fra loro.

Il modello di sensibilizzazione. Senza negare alla famiglia d'origine un ruolo centrale nell'avvicinamento del giovane all'attività politica, il modello di sensibilizzazione ne ridimensiona tuttavia la capacità di determinazione dei contenuti valoriali delle nuove generazioni. Che la socializzazione politica primaria non comporti un attaccamento indefettibile e duraturo ai valori appresi fa da premessa alla spiegazione dell'attivazione politica proposta indipendentemente da scienziati politici (Prewitt 1965; Putnam 1976) e sociologi (Merelman e King 1986). La socializzazione politica che avviene nell'ambiente familiare è nondimeno importante, in quanto tende a circoscrivere il fuoco degli interessi intellettuali dei figli e a trasmettere le competenze per il loro soddisfacimento. Laddove la famiglia d'origine è politicizzata, in particolare per la presenza di uomini politici nel suo seno, l'impegno politico entra «naturalmente» a far parte dell'orizzonte delle opzioni di commitment professionale prese in considerazione dai giovani (Prewitt 1965, 107-109). La politicizzazione familiare, insomma, serve a rendere l'attivismo politico un babitus: «il modello di sensibilizzazione asserisce che i genitori e altre figure salienti trasmettono con successo abitudini 
durevoli di attivismo politico ai futuri attivisti fin dall'infanzia» (Merelman e King 1986, 476). Diversi studi americani presentano risultati in linea con questo argomento (Marvick e Nixon 1961, 209; Salisbury 1965, 561; Clarke et al. 1975, 217).

In aggiunta a ciò, si ritiene che gli attivisti siano dotati di uno «stile di apprendimento politico particolarmente aperto e flessibile» (Merelman e King 1986, 476) che, secondo altri autori (Stone 1974, 102-103; Ziller et al. 1977), costituirebbe una componente centrale di quella «complessità cognitiva» caratteristica dell'uomo politico. Questo tratto della personalità consiste in una marcata sensibilità ad argomentazioni contrarie e a sfumature interpretative che trova espressione in posizioni articolate di fronte a questioni di rilevanza collettiva (Tetlock 1985). Non è improbabile, inoltre, che, in presenza di una complessità cognitiva superiore alla media, la disponibilità di fondo all'attivismo derivante da un'esposizione precoce alle questioni politiche in ambito familiare trovi un modus operandi adeguato alla pratica politica vera e propria.

Il modello di identificazione razionale. Come punto di partenza il modello di identificazione razionale assume che gli individui, specie in giovane età, adottino identità idonee alla massimizzazione del loro status futuro. Si suppone, inoltre, che la politicizzazione della famiglia e dell'ambiente formativo giochino un ruolo fondamentale per la «produzione» dei giovani attivisti politici. Purtuttavia, il modello mette fra parentesi i processi di trasmissione di norme e valori; dà invece rilievo alla presenza di configurazioni tipiche di opportunità quali antecedenti cruciali della scelta di una determinata carriera, tenendo conto della trinità delle risorse-chiave per il conseguimento di status: il capitale economico (in particolare, l'ereditabilità di mezzi di produzione), il capitale culturale (in ispecie, il possesso di credenziali d'istruzione), il capitale sociale (ossia, l'inserimento in relazioni interpersonali di reciprocità) ${ }^{6}$. Più nel dettaglio,

6 Visto che negli ultimi anni il concetto di «capitale sociale» è stato sottoposto a vigorosi «stiramenti concettuali» (cfr. Newton 1997), si precisa che il suo impiego in questo articolo è conforme all'accezione originaria proposta da Bourdieu, indicando «l'aggregato delle risorse attuali e potenziali che sono connesse al possesso di una rete durevole di relazioni più o meno istituzionalizzate di mutua conoscenza e riconoscenza»; in particolare, si assume che «il volume del capitale sociale posseduto da un dato attore dipende dalla dimensione della rete di legami che costui può effettivamente mobilitare e dal volume del capitale (economico, culturale o simbolico) posseduto per parte propria da ciascuno di coloro a cui è collegato» (Bourdieu 1986, 248-249). 
l'emergere dell'aspirazione giovanile ad intraprendere una carriera politica si ricostruisce come l'esito di un processo che si innesca all'approssimarsi dell'età adulta, allorché l'aspettativa sociale per una predefinizione degli orientamenti professionali individuali si fa più pressante.

In primo luogo, dunque, si ritiene che normalmente i giovani possano permettersi di dedicarsi ad attività politiche solo quando dispongano di una dotazione di base delle risorse principali che minimizzano i rischi di disoccupazione e sottoccupazione al momento di entrare nel mondo del lavoro: un'istruzione di livello universitario e opportunità professionali ascritte (derivanti dal controllo familiare di imprese o accessi privilegiati al lavoro). Credenziali d'istruzione e agevolazioni personali possono rivelarsi specialmente utili come rete di sicurezza per consentire un eventuale ingresso ritardato, a causa dell'impegno politico giovanile, in una carriera lavorativa.

In secondo luogo, si sottolinea che nei processi di riproduzione sociale le famiglie tendono a passare ai giovani adulti «le risorse chiave delle informazioni riservate sulle regole del gioco e i legami interpersonali» negli ambienti loro noti (Bertaux 1995,72 ). In quest'ottica, le famiglie politicizzate possono mettere a disposizione dei figli una conoscenza diretta di potenziali sponsor politici. Operativamente, ci si aspetta quindi che i giovani attivisti di partito abbiano un rapporto personale con qualche uomo politico fin da prima di entrare in politica.

A tale rapporto, infine, il modello attribuisce una valenza al contempo emotiva, sì che il titolare venga a giocare un ruolo di mentore ${ }^{7}$, e strumentale, in quanto valga ad assicurare risorse utili per svolgere attività politica. $\mathrm{Da}$ una parte, si assume che il sé consista di un insieme di identità sociali la cui diversa «salienza» è determinata dall'autorità di ciascun altro significativo con cui l'individuo si associa (Stryker 1981); il legame personale

7 Il concetto di «mentore» è ripreso dall'analisi di Levinson della transizione tra tarda adolescenza e gioventù: «Il mentore ha di solito diversi anni di più, è una persona di grande esperienza nel mondo in cui il giovane sta entrando. Nessuna parola corrente è adeguata a significare la natura della relazione che abbiamo in mente. Parole come "eminenza grigia" o "guru" suggeriscono i significati più sottili, ma hanno anche altre connotazioni che sarebbero fuorvianti. Il termine "mentore" è generalmente usato in un senso più ristretto, per riferirsi a un insegnante, un consigliere o uno sponsor. Per come usiamo il termine, esso significa tutte queste cose, e anche qualcosa di più» (Levinson 1978 , 97). Questa categoria è stata impiegata nella ricostruzione delle biografie di leader politici da Kellerman (1978) e Kearney (1984). 
con un uomo politico (di solito una persona relativamente importante nell'esperienza di un giovane) può quindi promuovere o amplificare un'identità politicamente orientata. D'altra parte, il mentore può contribuire a sviluppare, o comunque a piegare ad usi politici, una risorsa particolarmente rilevante per fare politica: un volume considerevole di capitale sociale, ossia un'ampia rete di relazioni, che è condizione generalmente necessaria per svolgere quelle attività di mediazione (tra rappresentanti politici e cittadini) e mobilitazione (in particolare del consenso) tipiche dell'attivista di partito.

\section{I dati}

Per cercare di mettere a fuoco - con le lenti delle ipotesi appena illustrate - cosa caratterizzi i giovani attivisti di partito rispetto alla generazione cui appartengono, si sono intervistati 115 dirigenti delle organizzazioni giovanili dei quattro partiti maggiori nel 1993 (Pds, Ppi, Psi, Lega Nord) ${ }^{8}$ e 322 individui della stessa coorte tra cui non figurano militanti di partito'. La gran parte dell'analisi verrà condotta sul campione risultante dalla combinazione di questi due campioni - quello degli attivisti e dei coetanei apolitici - secondo un disegno case-control (noto anche come campionamento response-based o choicebased $)^{10}$. Con questo disegno di ricerca è possibile studiare le

8 Il campione comprende anche quattro attivisti del movimento giovanile di $\mathrm{Al}$ leanza Democratica (Ad).

9 La composizione di questo campione è stata determinata da un'estrazione casuale dagli elenchi stradali delle provincie di Firenze e Pistoia (Recchi 1997a, 102-108). La sua struttura d'età è risultata essere complessivamente simile a quella del campione degli attivisti politici, seppure con una media anagrafica leggermente inferiore ( 23 anni contro 24 e mezzo per gli attivisti). L'unica deviazione significativa del gruppo di controllo dalla popolazione consiste nella sovrarappresentazione delle giovani donne (sono il $56 \%$ ); per il resto, tuttavia, va segnalata la sua quasi perfetta corrispondenza con il profilo nazionale della distribuzione delle famiglie su base di classe (il confronto è con $\mathrm{i}$ dati di Cobalti e Schizzerotto 1994, 72).

10 In termini operativi, si tratta di affrontare il seguente problema: «data l'esistenza di due popolazioni A e $B$; dato un campione casuale di individui che sappiamo provenire da $A$, e di un altro campione casuale che sappiamo provenire da B; come possiamo fissare una regola per attribuire ulteriori individui, della cui origine si è incerti, alla popolazione cui effettivamente appartiene?» (Kendall 1980, 145). L'analisi discriminante $\mathrm{e}$ la regressione logistica sono le due risposte statisticamente più adeguate al problema. Tuttavia, la regressione logistica si fa preferire in quanto non si fonda sugli assunti rigidi dell'analisi discriminante - cioè, l'eguaglianza delle matrici di covarianza dei gruppi e la distribuzione normale multivariata delle variabili esogene (Press e Wilson 1978, 
determinanti di fenomeni particolarmente rari laddove il campionamento casuale semplice non consentirebbe di prendere in considerazione un numero sufficiente di casi che presentano la modalità meno frequente della variabile dipendente.

\section{Le variabili}

Benché la collocazione su un'unica dimensione di azioni tanto diverse come il voto e la militanza di partito sollevi non poche riserve (Aldrich 1997), una tradizione politologica consolidata tratta l'attivismo come una forma di partecipazione politica. Perciò l'analisi empirica prenderà le mosse da un modello di base largamente adottato negli studi sulla partecipazione politi$\mathrm{ca}$, incentrato su fattori personali e familiari che rappresentano varie dimensioni di «centralità sociale»: il genere, l'occupazione, la posizione di classe della famiglia d'origine, l'area di residen$\mathrm{za}^{11}$. Da questo ineludibile punto di partenza si procederà al controllo dei modelli più specifici presentati nelle pagine precedenti.

Nel modello di compensazione, come si ricorderà, l'attivismo politico giovanile costituisce un modo per cercare di porre rimedio ad alcuni problemi adolescenziali - da un lato per sentirsi più integrati nella vita di un gruppo e nei rapporti con gli altri, dall'altro per accrescere il proprio senso di controllo sulla realtà. Quali indicatori di questi cambiamenti attesi si prendono tre variabili dicotomiche afferenti al senso di appartenenza ad un gruppo, al senso di socialità e al senso di controllo personale ${ }^{12}$.

705). L'analisi multivariata dei dati, quindi, si svilupperà tramite regressioni logistiche che applicano una stima di massima probabilità per campioni casuali. L'uso di questa tecnica con campioni response-based è considerata legittima dalla letteratura statistica; l'unica avvertenza è che i parametri dell'intercetta - che peraltro qui non interessano non possono essere interpretati nel modo consueto (cfr. Prentice e Pyke 1979; Xie e Manski 1989, 289).

11 Le formulazioni originarie del modello di centralità sociale rimontano a Lane (1959, 195-197) e Milbrath e Goel (1977, 87 e ss.). Secondo questi ultimi autori, che «le persone vicine al centro della società abbiano maggiori probabilità di partecipazione di quelle vicine alla periferia» costituisce «una delle proposizioni più minuziosamente provate nelle scienze sociali» (ibidem, 89 ). Alcune implicazioni e limiti del modello sono discussi in un ormai classico saggio di Pizzorno (1993, 114-120).

$12 \mathrm{Nel}$ questionario, $\mathrm{i}$ tre item erano i seguenti: «Pensa ora attentamente a come eri alcuni anni fa. Cosa è cambiato nella tua vita rispetto agli anni dell'adolescenza? 
Il modello di cristallizzazione postula che i giovani si attivino politicamente in ragione della loro adesione ad un'ideologia negli anni della socializzazione politica primaria. $\mathrm{Ci}$ si aspetta quindi che le configurazioni valoriali degli attivisti siano prossime a schemi ideologici, e, anche, che l'impegno politico postadolescenziale induca a ritenersi più coerenti con $\mathrm{i}$ valori in cui si crede di quanto non fosse in precedenza. Coerenza ideologica (oggettiva) e senso di coerenza (soggettivo) sono registrati rispettivamente da un indice e una variabile dicotomica ${ }^{13}$. Il modello inoltre prevede che una forte consonanza politica con familiari ed amici intimi faciliti la scelta di attivarsi politicamente, sia in via diretta che rendendo più cogente l'effetto causale della coerenza ideologica ${ }^{14}$.

Il modello di sensibilizzazione pone in primo piano la tradizione familiare di impegno politico, che è stata rilevata - e codificata in forma dicotomica - sulla base dell'esistenza di uno o più parenti in politica (almeno in qualità di attivisti di partito). Inoltre ci si aspetta che $i$ giovani attivisti abbiano una complessità cognitiva superiore ai coetanei apolitici ${ }^{15}$.

Secondo il modello di identificazione razionale l'attivazione politica giovanile richiede anzitutto il controllo di risorse di base per il conseguimento di status: almeno un'istruzione di li-

Oggi ti senti [più di allora/meno di allora/come allora?]: 1) Parte di un gruppo unito e con la sua identità; 2) Una persona con-molti amici; 3) Una persona con un futuro incerto» [l'ultimo item controscalato per evitare response-set]. In sede di analisi ciascuna variabile è stata dicotomizzata (più di allora/meno o come allora).

13 L'indice di coerenza ideologica è stato costruito, secondo la tecnica proposta da Barton e Parsons (1977), a partire da una cluster analysis di cinque scale di atteggiamenti («etnocentrismo», «radicalismo», «importanza del rispetto delle gerarchie», «importanza dell'autorità nell'educazione infantile», «opposizione all'aborto»). Si sono così individuati tre pattern di risposta tipici, corrispondenti ad orientamenti di valore di sinistra, di centro-cattolici e di destra, assunti come profili ideologici di fondo. Poiché la distanza degli intervistati dal profilo cui essi si mostrano più vicini rappresenta una misura del loro grado di deideologizzazione, si è considerato l'indice di coerenza ideologica pari all'inverso di tale distanza. La percezione di un mutamento nel senso di coerenza ideologica è invece colta con il seguente item: «Pensa ora attentamente a come eri alcuni anni fa. Cosa è cambiato nella tua vita rispetto agli anni dell'adolescenza? Oggi ti senti [più di allora/meno di allora/come allora?]: Coerente con un sistema di idee». In sede di analisi la variabile è stata dicotomizzata (più di allora/meno o come allora).

14 Come indicatore di consonanza politica familiare si assume la percentuale di familiari con le stesse preferenze partitiche dell'intervistato. Allo stesso modo è stato costruito l'indicatore di consonanza politica con gli amici intimi, definiti operativamente come quel sottoinsieme delle persone che l'intervistato dichiara di frequentare nel tempo libero e che conosce almeno dall'età di 16 anni (esclusi i familiari).

15 L'indice di complessità usato nell'analisi è pari al numero di risposte non polari sulle scale Likert del questionario (per un indice più articolato cfr. Recchi 1997a, 128-130). 
vello universitario ${ }^{16}$ o l'esistenza di opportunità di lavoro di origine familiare ${ }^{17}$. Si prevede che i giovani cui manchino entrambe queste risorse evitino di investire il loro tempo in attività politiche. In secondo luogo, si presuppone l'esistenza di parenti in politica, che possono direttamente fungere da sponsor o introdurre l'aspirante attivista a un potenziale sponsor non familia$\mathrm{re}^{18}$, rendendo costui sensibile alle sorti del giovane. Infine, il modello chiama in causa la disponibilità di un ampio volume di capitale sociale - una risorsa proficua per la mobilitazione del consenso e per altre attività di mediazione politica. Come suo indicatore si ricorre al numero di conoscenti degli intervistati al momento della rilevazione ${ }^{19}$. Sebbene a rigore descriva una situazione successiva all'attivazione politica, questo dato non cresce in misura significativa all'aumentare dell'età di coinvolgimento nelle attività di partito e per il fatto di aver ricoperto cariche pubbliche elettive (nonché sulla base di altri possibili fattori condizionanti: cfr. tab. 1). Pare dunque legittimo ritenere che possa dar conto in modo accettabile delle differenze preesistenti nel volume di capitale sociale detenuto dagli attivisti.

16 La variabile è dicotomica, a seconda che gli intervistati siano iscritti all'università (o abbiano conseguito la laurea, se hanno più di 25 anni) o meno. Per i giovani attivisti ci si basa sul loro livello d'istruzione al momento in cui hanno cominciato a far politica attivamente.

17 Quest'ultimo fattore è operativizzato in forma dicotomica sulla base della condizione lavorativa (autonoma o dipendente) del padre, assumendo che i figli di imprenditori o lavoratori indipendenti possono affiancare o rimpiazzare $\mathrm{i}$ genitori valendosi di quanto da loro già accumulato per il proseguimento dell'attività. Occorre notare che, per quanto l'ereditarietà paterna costituisca il filone principale di trasmissione delle opportunità occupazionali per via familiare, talora entrano in gioco anche rami secondari della parentela che non sono tenuti in conto dall'indicatore impiegato.

18 I legami con possibili sponsor politici sono stati registrati con tecniche leggermente diverse nelle interviste con i giovani apolitici e con gli attivisti. Nel primo caso, si è chiesto se essi conoscessero personalmente «un dirigente di partito, un amministratore locale, un parlamentare» (tre item distinti). A ciascun attivista, invece, si è domandato se vi fossero «persone che fanno politica, e che ritieni più importanti ed esperte, con cui ti capita di intrattenerti con una certa consuetudine per chiedere consiglio». Oltre ad approfondire le caratteristiche personali di queste persone, si è chiesto da quanti anni l'intervistato le conoscesse. Le relazioni con costoro sono state codificate come relazioni di sponsorship solo se risalivano a prima dell'ingresso in politica del giovane.

19 Per la precisione, la variabile registra il numero di individui inclusi da ciascun intervistato sotto la lettera «D» della propria agenda telefonica personale (per vantaggi e limiti di questa procedura, cfr. Recchi 1997a, 170-171). 
TAB. 1. Le origini del volume di capitale sociale dei giovani attivisti di partito: regressione lineare

\begin{tabular}{|c|c|c|}
\hline Variabili indipendenti & (b) & E.s. \\
\hline Età & $-0,004$ & 0,046 \\
\hline Genere $=$ maschio & $-0,327$ & 0,255 \\
\hline Occupazione $=$ ha un lavoro & 0,142 & 0,250 \\
\hline Classe sociale familiare $=$ borghesia $^{a}$ & $-0,128$ & 0,394 \\
\hline Classe sociale familiare $=$ classe media impiegatizia ${ }^{a}$ & $-0,146$ & 0,380 \\
\hline Classe sociale familiare $=$ piccola borghesia indipendente ${ }^{a}$ & 0,536 & 0,418 \\
\hline Luogo di residenza $=$ capoluogo di provincia & 0,369 & 0,216 \\
\hline Età all'ingresso in politica & $-0,076$ & 0,042 \\
\hline Elezione a carica pubblica & 0,094 & 0,273 \\
\hline Partito $=\mathrm{Ppi}^{b}$ & 0,427 & 0,382 \\
\hline$=\mathrm{Pds} b$ & 0,219 & 0,415 \\
\hline$=$ Lega Nord $b$ & $-0,065$ & 0,491 \\
\hline Costante & 1,852 & 1,271 \\
\hline
\end{tabular}

a Categoria di riferimento: Classe operaia.

$b$ Categoria di riferimento: Psi-Ad.

$\mathrm{N}=102$. Gradi di libertà $=12 . \mathrm{R}^{2}=0,023$.

\section{Il controllo empirico dei modelli}

La tabella 2 presenta sette regressioni logistiche della variabile dipendente - l'attivismo politico degli intervistati (codificato «1» per i giovani attivisti di partito, «0» per i coetanei apolitici) - sugli indicatori dei modelli fin qui illustrati secondo una strategia analitica di saturazione progressiva (nelle prime cinque regressioni tenendo conto degli effetti principali, nelle ultime due inserendo anche le interazioni teoricamente rilevanti). Tale strategia consente di apprezzare come, allorché si introducono via via altri fattori, l'effetto di alcune variabili indipendenti prima facie significative finisca per ridursi o scompaia ${ }^{20}$.

$\mathrm{E}$ il caso, in particolare, della variabile «classe», che costi-

20 È opportuno ricordare che nelle regressioni logistiche i valori assunti dai parametri che esprimono gli effetti esercitati da ciascuna variabile indipendente rappresentano le variazioni nel logaritmo della quota di probabilità (odd) che la variabile dipendente dicotomica abbia il valore corrispondente alla categoria di riferimento (in questo caso «1», con cui si indica la proprietà di essere attivisti di partito) all'aumentare di una unità del valore della medesima variabile indipendente, tenendo sotto controllo tutte le altre variabili indipendenti incluse nell'equazione di regressione. È necessario anche segnalare che $i$ parametri non sono standardizzati, per cui i loro valori non risultano immediatamente comparabili fra loro. 
tuisce l'architrave del modello di centralità sociale ${ }^{21}$. È sì vero che le probabilità di avere a che fare con giovani attivisti sono tanto più alte quanto più si sale nella gerarchia delle classi, confermando - ancora una volta - che «chi ha maggiori risorse socio-economiche partecipa più di chi ne ha meno» (Verba et al. $1978,1)^{22}$. Ed è anche vero che le caratteristiche psicologiche (regressioni 2, 3 e 4) non modificano sostanzialmente queste probabilità. Tuttavia, l'effetto delle differenze di classe viene meno non appena si prendono in esame (regressioni 5, 6 e 7) le variabili che operativizzano il modello di identificazione razionale. Vi è da supporre che queste variabili incorporino i meccanismi cruciali tramite i quali le posizioni di classe condizionano la scelta di attivarsi politicamente in giovane età. In altri termini, il modello di identificazione razionale pare decomporre l'effetto della collocazione di classe nei suoi elementi direttamente influenti per l'assunzione di un impegno politico - il legame con uno sponsor, un vasto capitale sociale, una dotazione minima di risorse d'istruzione o di risorse economiche familiari per ammortizzare il costo di tale impegno.

L'esistenza di forti differenze di genere è invece in linea con il modello di centralità sociale ${ }^{23}$. Nessuno degli altri modelli dà rilievo esplicito a questo fattore. Che esso eserciti un effetto sempre e comunque significativo getta un'ombra su quelle interpretazioni della scarsa presenza di donne in politica che imputano il fenomeno alla scarsa presenza di donne fra le categorie sociali da cui provengono tendenzialmente gli attivisti politici (per esempio, Darcy et al. 1987, 96). I dati mostrano che le giovani non rimangono lontane dall'impegno politico perché (o soltanto perché) difettano delle risorse preliminari all'autoreclutamento, ma anche per il mero fatto di essere (considerate e considerarsi) donne. Forse, non è fuori luogo una spiegazione ad hoc che rinvia a specificità della nostra cultura politica, dato

21 Secondo Milbrath e Goel $(1977,92)$, le differenze di classe nella partecipazione politica sono tanto spiccate da emergere «indipendentemente da come si misurano (sic) le classi». Per quest'analisi, si è adottata una classificazione quadritomica della classe sociale della famiglia dell'intervistato ispirata allo schema di Erikson e Goldthorpe (1992, 35 e ss.).

22 Come osservano talora gli stessi studiosi della partecipazione politica che ne propugnano un'analisi unidimensionale, le differenze di classe si rivelano empiricamente tanto più marcate «allorché si distingue fra l'atto di votare e atti politici "più difficili"» - come la militanza di partito, per l'appunto (Brady et al. 1995, 290).

23 Occorre anche notare che quest'effetto è forse parzialmente enfatizzato dalla sovrarappresentazione delle donne nel campione di controllo. 
ТАВ. 2. Le radici dell'attivismo giovanile di partito: regressioni logistiche

\begin{tabular}{|c|c|c|c|c|c|c|c|c|c|c|c|c|c|c|}
\hline \multirow[b]{2}{*}{ Variabili indipendenti } & \multicolumn{2}{|l|}{1} & \multicolumn{2}{|l|}{2} & \multicolumn{2}{|l|}{3} & \multicolumn{2}{|l|}{4} & \multicolumn{2}{|l|}{5} & \multicolumn{2}{|l|}{6} & \multicolumn{2}{|l|}{7} \\
\hline & (b) & E.s. & (b) & E.s. & (b) & E.s. & (b) & E.s & (b) & E.s. & (b) & E.s. & (b) & E.s. \\
\hline $\begin{array}{l}\text { Genere }=\text { maschile } \\
\text { Occupazione }=\text { ha un lavoro } \\
\text { Classe sociale familiare }=\text { borghesia } a \\
\text { Classe sociale familiare }=\text { classe media impiegatizia } \\
\text { Classe sociale familiare }=\text { piccola borghesia indipendente } \\
\text { Luogo di residenza }=\text { capoluogo di provincia }\end{array}$ & $\begin{array}{l}1,682^{* *} \\
0,104 \\
2,777^{* *} \\
1,998^{* *} \\
0,956^{*} \\
0,195\end{array}$ & $\begin{array}{l}0,285 \\
0,278 \\
0,449 \\
0,407 \\
0,427 \\
0,266\end{array}$ & $\begin{array}{ll}5 & 1,687^{* *} \\
8 & 0,200 \\
9 & 3,093^{* *} \\
7 & 2,163^{* *} \\
7 & 0,922^{*} \\
6 & 0,093\end{array}$ & $\begin{array}{l}0,307 \\
0,298 \\
0,483 \\
0,436 \\
0,448 \\
0,285\end{array}$ & $\begin{array}{l}1,819^{* *} \\
0,327 \\
3,060^{* *} \\
2,252^{* *} \\
0,929^{*} \\
0,081\end{array}$ & $\begin{array}{l}0,336 \\
0,330 \\
0,509 \\
0,465 \\
0,473 \\
0,315\end{array}$ & $\begin{array}{l}1,842^{* *} \\
0,241 \\
2,380^{* *} \\
1,637^{* *} \\
1,079 \\
0,077\end{array}$ & $\begin{array}{l}0,457 \\
0,439 \\
0,680 \\
0,618 \\
0,608- \\
0,430\end{array}$ & $\begin{array}{c}1,564^{* *} \\
0,748 \\
1,227 \\
0,996 \\
-0,512 \\
0,009\end{array}$ & $\begin{array}{l}0,545 \\
0,617 \\
0,859 \\
0,754 \\
0,821 \\
0,542\end{array}$ & $\begin{array}{l}1,832^{\star *} \\
0,838 \\
1,117 \\
1,021 \\
0,003 \\
0,110\end{array}$ & $\begin{array}{l}0,586 \\
0,659 \\
0,926 \\
0,808 \\
0,862 \\
0,579\end{array}$ & $\begin{array}{l}1,932^{* * *} \\
1,034 \\
0,991 \\
0,864 \\
0,029 \\
0,486\end{array}$ & $\begin{array}{l}0,617 \\
0,704 \\
0,960 \\
0,846 \\
0,906 \\
0,629\end{array}$ \\
\hline $\begin{array}{l}\text { Senso di controllo personale = più che nell'adolescenza } \\
\text { Senso di appartenenza a gruppo = più che nell'adolescenza } \\
\text { Senso di socialità = più che nell'adolescenza }\end{array}$ & & & $\begin{array}{l}-0,722^{*} \\
1,590^{* *} \\
-0,355\end{array}$ & $\begin{array}{l}0,369 \\
0,286 \\
0,277\end{array}$ & $\begin{array}{l}-0,897^{*} \\
1,835^{* *} \\
-0,545\end{array}$ & $\begin{array}{l}0,395 \\
0,330 \\
0,301\end{array}$ & $\begin{array}{l}-0,153 \\
1,898^{* *} \\
-0,306\end{array}$ & $\begin{array}{l}0,465 \\
0,428 \\
0,405\end{array}$ & $\begin{array}{l}-0,253 \\
1,803 * * \\
-0,931\end{array}$ & $\begin{array}{l}0,564 \\
0,521 \\
0,522\end{array}$ & $\begin{array}{l}-0,291 \\
1,612^{*} \\
-0,493\end{array}$ & $\begin{array}{l}0,997 \\
0,646 \\
0,619\end{array}$ & $\begin{array}{c}-0,288 \\
1,389^{*} \\
-0,299\end{array}$ & $\begin{array}{l}1,022 \\
0,676 \\
0,648\end{array}$ \\
\hline $\begin{array}{l}\text { Senso di coerenza = più che nell'adolescenza } \\
\text { Coerenza ideologica } \\
\text { Consonanza politica con familiari } \\
\text { Consonanza politica con amici intimi }\end{array}$ & & & & & $\begin{array}{r}-0,569 \\
0,137 \\
1,676^{* *} \\
1,223^{* *}\end{array}$ & $\begin{array}{l}0,312 \\
0,149 \\
0,437 \\
0,440\end{array}$ & $\begin{array}{l}-0,438 \\
0,256 \\
1,323^{* *} \\
1,099^{*}\end{array}$ & $\begin{array}{l}0,415 \\
0,209 \\
0,553 \\
0,566\end{array}$ & $\begin{array}{c}-0,146 \\
0,191 \\
1,496^{*} \\
0,332\end{array}$ & $\begin{array}{l}0,526 \\
0,253 \\
0,708 \\
0,698\end{array}$ & $\begin{array}{l}-0,069 \\
-0,015 \\
1,559 * \\
0,694\end{array}$ & $\begin{array}{l}0,547 \\
0,553 \\
0,755 \\
0,766\end{array}$ & $\begin{array}{l}0,223 \\
0,085 \\
1,380 \\
0,918\end{array}$ & $\begin{array}{l}0,578 \\
0,589 \\
0,768 \\
0,791\end{array}$ \\
\hline $\begin{array}{l}\text { Parente in politica } \\
\text { Complessità cognitiva }\end{array}$ & & & & & & & $\begin{array}{l}4,132^{\text {** }} \\
-0,157\end{array}$ & $\begin{array}{l}0,536 \\
0,203\end{array}$ & $\begin{array}{l}4,811^{* *} \\
-0,188\end{array}$ & $\begin{array}{l}0,697 \\
0,258\end{array}$ & $\begin{array}{l}5,247^{* * *} \\
-0,168\end{array}$ & $\begin{array}{l}1,185 \\
0,308\end{array}$ & $\begin{array}{l}4,686^{* *} \\
-0,196\end{array}$ & $\begin{array}{l}0,778 \\
0,322\end{array}$ \\
\hline $\begin{array}{l}\text { Risorse di base per il conseguimento di status = disponibili } \\
\text { Legame preesistente con sponsor politico non familiare } \\
\text { Volume del capitale sociale }\end{array}$ & & & & & & & & & $\begin{array}{l}1,405^{*} \\
1,545^{* *} \\
0,221^{* *}\end{array}$ & $\begin{array}{l}0,690 \\
0,553 \\
0,041\end{array}$ & $\begin{array}{l}1,493^{*} \\
0,812 \\
0,184^{* *}\end{array}$ & $\begin{array}{l}0,744 \\
0,959 \\
0,062\end{array}$ & $\begin{array}{c}1,617^{*} \\
-0,038 \\
0,055\end{array}$ & $\begin{array}{l}0,789 \\
0,787 \\
0,067\end{array}$ \\
\hline $\begin{array}{l}\text { Senso di controllo personale } x \text { Senso di appartenenza a gruppo } \\
\text { Senso di controllo personale } x \text { Senso di socialità } \\
\text { Consonanza politica con familiari } x \text { Coerenza ideologica } \\
\text { Consonanza politica con amici intimi } x \text { Coerenza ideologica } \\
\text { Parente in politica } x \text { Complessità cognitiva } \\
\text { Parente in politica } x \text { Volume del capitale sociale } \\
\text { Sponsor non familiare } x \text { Volume del capitale sociale } \\
\text { Parente in politica o Sponsor non familiare } x \\
\text { Volume del capitale sociale }\end{array}$ & & & & & & & & & & & $\begin{array}{r}1,040 \\
-1,308 \\
0,861 \\
-0,570 \\
-0,565 \\
-0,060 \\
0,076\end{array}$ & $\begin{array}{l}1,170 \\
1,227 \\
0,752 \\
0,929 \\
0,719 \\
0,136 \\
0,084\end{array}$ & $\begin{array}{r}1,111 \\
-1,720 \\
0,717 \\
-0,601 \\
-0,603\end{array}$ & $\begin{array}{l}1,220 \\
1,264 \\
0,787 \\
1,020 \\
0,744\end{array}$ \\
\hline $\begin{array}{l}\text { Costante } \\
\chi^{2} \\
\text { Gradi di libertà }\end{array}$ & $\begin{array}{r}-3,714 \\
118 \\
6\end{array}$ & 0,433 & $\begin{array}{r}3-142 \\
156 \\
9\end{array}$ & 0,518 & $\begin{array}{r}-5,614 \\
196 \\
13\end{array}$ & $\begin{array}{l}0,666 \\
6,6 \\
3\end{array}$ & $\begin{array}{r}-6,391 \\
293 \\
15\end{array}$ & $\begin{array}{l}0,874 \\
3^{3}\end{array}$ & $\begin{array}{r}-9,189 \\
354 \\
18\end{array}$ & $\begin{array}{l}1,394 \\
4,2 \\
8\end{array}$ & $\begin{array}{r}-9,492 \\
358 \\
25\end{array}$ & $\frac{1,527}{3,4}$ & $\begin{array}{r}-9,278 \\
367 \\
24\end{array}$ & $4^{1,537}$ \\
\hline
\end{tabular}

* $p<0,05 ; * * x<0,01$.

a Categoria di riferimento: Classe operaia.

$\mathrm{N}=422$. 
che in prospettiva comparata è noto che la componente femminile della classe politica - a tutti i livelli - è minore in Italia che nella maggior parte dei paesi europei (Guadagnini 1993, 175 e ss.). Ma torneremo sul punto più avanti.

Non di rado si assume che le esperienze lavorative promuovano la politicizzazione degli individui sia come veicolo di rapporti organizzativi con gruppi contigui ai partiti, quali i sindacati e le associazioni di categoria, sia come stimolo ad una presa di coscienza dei propri interessi. In sintonia con quest'ultima chiave di lettura, la scarsa politicizzazione dei giovani non ancora entrati nel mondo del lavoro viene spesso attribuita all'incostanza e alla dispersione dei loro «reali interessi» (ad esempio, Hudon e Hébert 1994, 370). D'altro canto, i giovani che non lavorano - specie se studenti - dispongono di una risorsa estremamente preziosa per l'impegno politico: il tempo libero (Milbrath e Goel 1977, 116). Ė possibile che questi incentivi all'attivazione politica (il consolidamento dei propri interessi e il tempo disponibile) in qualche misura si controbilancino; di conseguenza, la condizione lavorativa, sebbene positivamente correlata con l'attivismo di partito, non ne costituisce un fattore predittivo significativo. Così come non è affatto significativo vivere in un contesto urbano, forse anche in virtù del radicamento territoriale diffuso dei partiti italiani (quantomeno al momento della rilevazione).

A partire dalla seconda equazione di regressione, l'analisi si estende alle variabili che operativizzano il modello di compensazione. Ancorché parzialmente significativi, i loro parametri si rivelano teoricamente incongrui. Contrariamente alle attese, infatti, il senso di controllo personale degli attivisti di partito è diminuito anziché crescere rispetto agli anni dell'adolescenza. Ad ogni modo, l'effetto di questo fattore si riduce considerevolmente e perde significatività nelle regressioni $4,5,6$ e 7 . Anche l'esperienza soggettiva di un incremento di socialità esercita un impatto negativo (e comunque mai significativo) sull'attivismo. Sempre significativo risulta invece il coefficiente del senso di appartenenza ad un gruppo. Ma questo indicatore, da solo, non pare sufficiente a sostenere alcuna ipotesi. Senza il conforto degli altri indicatori del modello di compensazione, esso segnala nient'altro che un truismo - e cioè che i giovani che partecipano alla vita di gruppi organizzati (quali sono i partiti) tendono ad avere maturato sentimenti di appartenenza ad un gruppo più forti della media dei loro coetanei, che generalmente non sono altrettan- 
to integrati in strutture associative. Infine, nel modello di compensazione si contempla la possibilità che modifiche positive nella stima di sé post-adolescenziale (in particolare, un aumento del senso di controllo personale) possano influenzare la propensione ad attivarsi politicamente allorché siano accompagnate dal superamento di deficit derivanti da una scarsa integrazione sociale (indicati da un incremento nel senso di appartenenza di gruppo e di socialità). Tuttavia, le interazioni fra tali fattori, nelle regressioni 6 e 7, mostrano di non influire minimamente sull'attivazione politica. Nel complesso, l'argomento per cui l'ingresso in politica rappresenta un modo per aumentare la propria considerazione di sé non sembra empiricamente sostenibile. Nella migliore delle ipotesi, se ne deve rivedere la portata, limitandola ai casi di leader di primo piano (cfr. Knutson 1973, 50).

Nella terza regressione si introducono gli indicatori del modello di cristallizzazione. Uno di essi - l'incremento del senso soggettivo di coerenza ideologica - esercita un'influenza minima e negativa (al contrario di quanto supposto) e un altro - l'indice di coerenza ideologica - è solo molto blandamente associato con l'attivismo di partito; per contro, la consonanza con familiari ed amici intimi costituisce in prima battuta un elemento significativamente caratterizzante i giovani attivisti di partito. La rilevanza empirica di queste ultime due variabili era emersa anche in uno studio americano in cui si sottolineava che «quei giovani che hanno un'affiliazione di partito e preferenze elettorali simili ai loro genitori e amici hanno maggiori possibilità di diventare attivisti» (Merelman e King 1986, 486-487). Nel nostro caso, tuttavia, dopo aver controllato anche gli indicatori del modello di identificazione razionale (nelle regressioni 5, 6 e 7), solo la sintonia politico-partitica con i familiari resta una componente causale significativa dell'attivismo giovanile. Ma a questo consenso nel sostegno a un partito non fanno riscontro configurazioni valoriali particolarmente definite. Le difficoltà del modello di cristallizzazione, in buona sostanza, discendono dal suo incardinamento nel dibattuto primacy principle della socializzazione politica, secondo cui i valori politici appresi in tenera età resterebbero pressoché inalterati in seguito (Marsh 1971; Searing et al. 1973; Searing et al. 1976). Questo principio teorico sembra valere ancor meno per gli attivisti politici (Dowse e Hughes 1977; Renshon 1989).

L'introduzione nell'analisi dei due indicatori del modello di sensibilizzazione migliora alquanto la capacità di rendere conto 
delle differenze tra i giovani attivisti e i coetanei apolitici. In particolare, avere un parente che fa politica accresce sensibilmente le probabilità per un giovane di entrare a far parte attiva di un'organizzazione di partito. Purtuttavia, dato che l'indice di complessità cognitiva si rivela del tutto ininfluente, come pure l'interazione fra complessità cognitiva e presenza di un parente in politica (regressioni 6 e 7), la combinazione di condizioni causali previste dal modello di sensibilizzazione si rivela priva di potenziale esplicativo quale che sia la sua specificazione statistica nell'equazione di regressione.

Il ruolo della politicizzazione familiare quale prerequisito dell'attivazione politica giovanile sembra trovare una cornice di riferimento empiricamente più solida nel modello di identificazione razionale. Non solo tutti gli indicatori di tale modello - avere un parente in politica, risorse ascrittive e/o d'istruzione di base, legami di vecchia data con potenziali sponsor politici e una vasta rete di conoscenze - risultano essere predittori significativi dell'attivismo di partito (regressione 5), ma, una volta che li si introduce nell'analisi, perdono importanza variabili come la collocazione di classe, la consonanza politica con gli amici e quella con i familiari (che rimane significativa, ma ad un livello inferiore).

Le regressioni 6 e 7 , che includono i fattori d'interazione teoricamente opportuni fra gli indicatori del modello di identificazione razionale, consentono di mettere meglio a fuoco alcuni punti. A prima vista (regressione 6), la rilevanza del volume di capitale sociale come predittore di attivismo politico giovanile non sembra dipendere dall'esistenza di familiari politicizzati o di sponsor esterni alla cerchia parentale, in quanto nessuno di questi due fattori - presi singolarmente - interagisce in modo significativo con la variabile in questione, mentre resta significativo il suo effetto diretto. Tuttavia, l'ipotesi prevedeva che il volume del capitale sociale influisse sull'attivismo politico giovanile in presenza dell'uno ovvero l'altro tipo di sponsor - cioè, indipendentemente dalla parentela con lo sponsor. Per render conto di ciò, si è costruito (regressione 7) un fattore d'interazione in cui si assume che l'effetto del volume del capitale sociale è condizione dell'esistenza di un familiare in politica o di uno sponsor partitico non familiare, o di entrambi ${ }^{24}$. Questo fattore

24 Sebbene in genere le interazioni fra variabili siano espresse in termini moltiplicativi, è noto che «altre forme funzionali possono essere dettate dalla teoria» (Jaccard et al. 1990, 78). L'interazione a tre variabili (chiamiamole X, W e Z) introdotta nell'ultima 
è decisamente significativo e azzera l'effetto diretto del volume di capitale sociale. Ciò impone di rivedere la conclusione di precedenti studi sulla partecipazione associativa secondo cui «la gente diventa più o meno attiva a seconda del numero di legami sociali di cui dispone» (Janoski e Wilson 1995, 272). Nel nostro caso, il possesso di una vasta rete di relazioni sociali interviene in maniera rilevante nel processo di attivazione politica giovanile soltanto allorché, in virtù di una relazione privilegiata con una figura politica di riferimento, vi si può fare ricorso per fini politicamente produttivi.

D'altro canto, la variabile "parente in politica" resta decisamente significativa anche in presenza di queste interazioni. Il perdurare del suo effetto causale indipendente sembra attestare che, nel processo di attivazione politica giovanile, il ruolo dei familiari politicizzati non può essere confinato al suo aspetto strumentale descritto dai fattori d'interazione - cioè, far fruttare politicamente una vasta rete di relazioni. Se, una volta controllate tutte le altre variabili, a monte dell'attivismo di partito in giovane età si registra l'importanza residua di una o più figure familiari che hanno già maturato un'esperienza in politica e la cui identità è in qualche modo segnata dalla vita di partito, si deve concludere che nel processo di attivazione interviene un elemento aggiuntivo, non rilevato in termini quantitativi, ma che si può ritenere consista in un incitamento o un sostegno emotivo all'impegno politico conforme alle aspettative del modello di identificazione razionale (Recchi 1997a, 178-180).

Nel complesso, l'analisi dei dati mostra la presenza contemporanea di elementi di razionalità e di identificazione che presiedono alla scelta di dedicarsi all'attività politica. La scelta è razionale nella misura in cui dipende dal controllo di credenziali d'istruzione e opportunità professionali ascrittive che ammortizzano il rischio di sovrainvestire in un'attività che offre prospettive incerte per la massimizzazione dello status futuro di un giovane. $\grave{E}$ razionale, inoltre, in quanto fa leva sul rapporto personale con possibili sponsor politici (familiari o meno), che possono moltiplicare le chances di carriera nel partito e le risorse relazionali utili, in ultima analisi, per guadagnare consenso. La scel-

riga delle regressione 7 è la seguente: $(\mathrm{X}$ or $\mathrm{W}) \mathrm{Z}$; cioè, passando dall'operatore logico ad operatori aritmetici: $(X+W-X W) Z$. Dato che $\mathrm{X}$ e W (ossia, avere un parente in politica e un potenziale sponsor non familiare) sono dicotomie, la loro interazione è pari a 0 solo quando entrambi hanno valore 0 ; altrimenti, l'interazione è pari a 1 . 
ta di far politica, tuttavia, non si esaurisce in queste premesse, richiedendo anche meccanismi di adesione psicologica, da intendere in termini di identificazione con un mentore - il che può spiegare perché avere un parente in politica, al netto dell'influenza di tutti gli altri fattori considerati, aumenta straordinariamente le probabilità che un giovane, almeno nell'Italia della prima metà degli anni novanta, si dedichi all'attività di partito.

\section{Attivisti, partecipativi e apatici: un'analisi supplementare}

Un ulteriore esame degli stessi dati consente di approfondire alcune questioni rimaste aperte. Per questa seconda analisi, il gruppo di controllo è stato spaccato in due sottogruppi in base al grado di coinvolgimento politico degli intervistati: $\mathrm{i}$ «partecipativi» (caratterizzati dall'aver preso parte nei sei mesi precedenti l'intervista ad almeno un'assemblea pubblica, una dimostrazione o una campagna di propaganda politica) e gli «apatici» (tutti gli altri). I partecipativi, nella fattispecie, costituiscono un segmento di popolazione giovanile che, sebbene non formalmente integrato in ruoli partitici, alla politica presta un'attenzione costante e anche qualche energia. Cosa impedisce loro di saltare il fosso che li separa dall'ingresso nei ranghi di un partito? E, d'altra parte, cosa tiene i giovani apatici alla larga da qualsiasi azione a contenuto politico? Per cercare di rispondere a queste domande è utile confrontare - nel campione in esame - gli effetti esercitati dalle variabili indipendenti sulle probabilità di dedicarsi alle attività di partito anziché, rispettivamente, non partecipare affatto o impegnarsi soltanto in ambito extrapartitico (tab. 3).

Il primo risultato da sottolineare ci riporta al tema delle differenze di genere. Una volta controllati gli altri fattori, la connotazione maschile degli attivisti risalta in maniera più netta rispetto ai giovani apatici anziché rispetto ai coetanei partecipativi. Dunque, il diaframma che separa le giovani donne dalla partecipazione politica sembra situarsi più nel passaggio dall'apatia ai comportamenti politici meno impegnativi che non nella eventuale assunzione di incarichi di partito. Poiché per intervenire ad assemblee, prender parte a dimostrazioni o collaborare ad una campagna elettorale di solito non è necessario sottoporsi ad alcuna forma di selezione esterna (potenzialmente discriminatoria), si deve ritenere che la componente autoselettiva - forse derivante 
TAB. 3. Effetto degli indicatori dei modelli interpretativi sul livello di coinvolgimento politico: regressioni logistichea

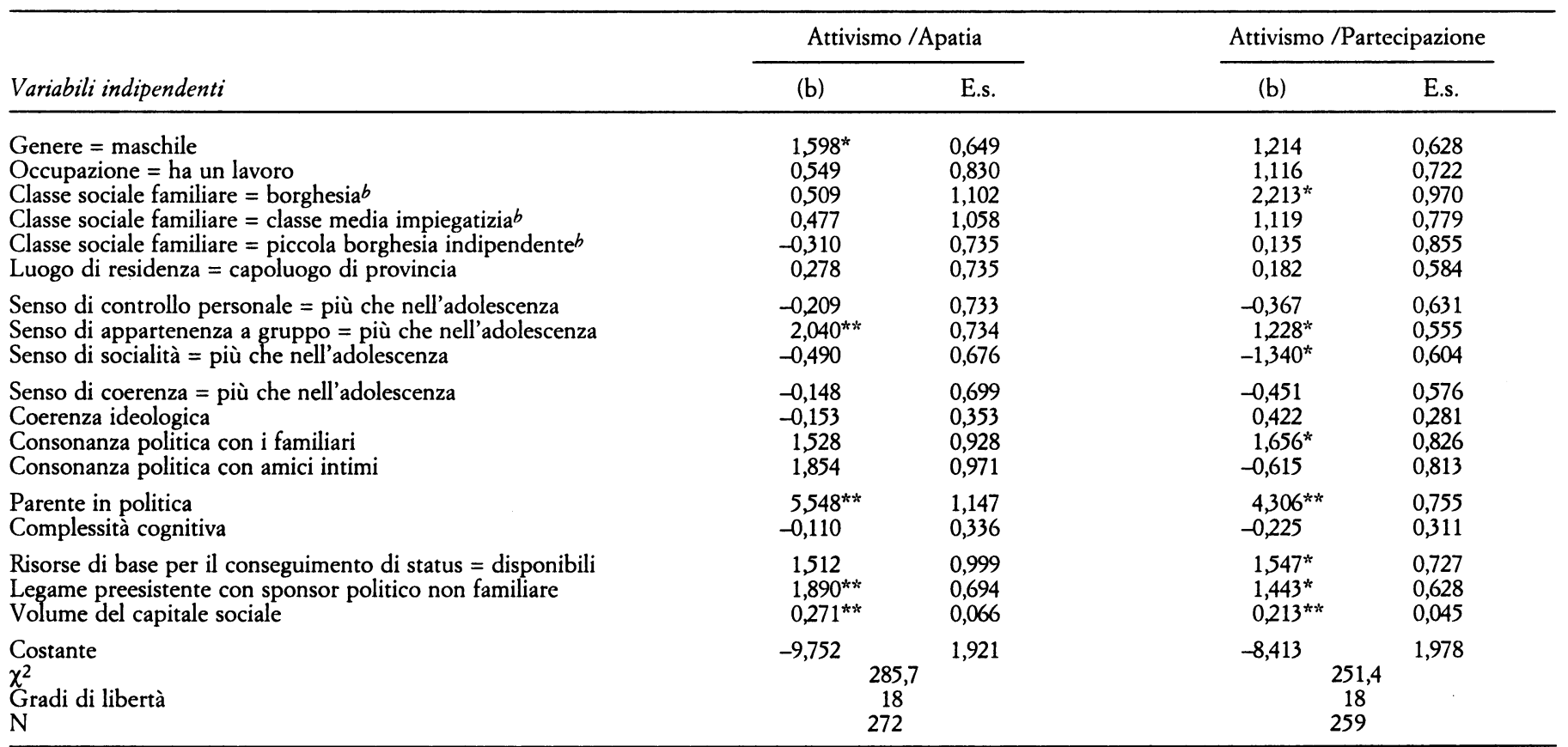

${ }^{*} \mathrm{p}<0,05 ;{ }^{* *} \mathrm{p}<0,01$.

a I parametri sono stati stimati con modelli separati di regressioni logistiche dicotomiche, vista l'equivalenza di efficacia rispetto alla stima di un unico modello politomico (cfr. Begg e Gray 1984).

$b$ Categoria di riferimento: Classe operaia. 
da modelli di socializzazione differenziati su base di genere giochi un ruolo prevalente nel minor coinvolgimento politico delle ragazze.

Nel complesso, i giovani partecipativi paiono essere più vicini ai coetanei apolitici che non agli attivisti di partito. Rispetto a questi ultimi, a partecipativi e apolitici fanno egualmente difetto quelle risorse specifiche previste dal modello di identificazione razionale. Peraltro, ai partecipativi la militanza di partito risulta essere pregiudicata in misura significativa anche da una collocazione di classe troppo bassa (il $51 \%$ sono figli di operai) e da una maggiore incertezza circa il proprio destino professionale (il $60 \%$ sono ancora studenti). Tra i partecipativi, infine, si contano relativamente pochi giovani che si dicono in sintonia con le preferenze politiche familiari, mentre si rivelano più numerosi della media coloro che condividono gli orientamenti politici degli amici ${ }^{25}$. Forse anche questa dissonanza degli atteggiamenti espressi nelle relazioni primarie non è estranea alla scelta di praticare forme intermittenti e non strutturate di partecipazione politica (cfr. Lane 1959, 108; Pizzorno 1993, 119).

In chiave teorica, questi risultati impongono una riflessione di portata più generale. Su molte dimensioni rilevanti (in ispecie l'appartenenza di classe, la condizione occupazionale, la consonanza con le preferenze politiche dei familiari) i giovani partecipativi non si collocano a metà strada fra gli apatici e gli attivisti, come invece dovrebbe essere se la partecipazione politica costituisse un'unica dimensione omogenea che riunisse comportamenti differenziati soltanto in ragione di una maggiore o minore intensità. Quantomeno nel nostro caso, non sembra dunque legittimo adottare un modello di spiegazione unitario valido per forme diverse di partecipazione. A questa conclusione, d'altra parte, di recente si è avvicinato persino chi ha tradizionalmente difeso una visione unidimensionale della partecipazione, ammettendo che «criteri decisionali differenti assumono rilievo in fasi distinte del processo di partecipazione in ambito politico» (Verba et al. 1997, 33). Forse è un primo passo verso il riconoscimento del fatto che azioni come votare, partecipare occasionalmente ad una manifestazione di piazza e contribuire in modo regolare alla vita di un partito rispondono a scelte in-

25 È inoltre significativa - a confronto con quella degli attivisti - la quota di partecipativi (il $56,6 \%$ ) che ritiene che in tempi recenti sia aumentata la cerchia dei propri amici. $\grave{E}$ possibile che la partecipazione politica fuori dai partiti sia motivata da esigenze di socialità che, per contro, la vita di partito non è in grado di soddisfare. 
dividuali incommensurabili e quindi richiedono, anziché una sola grande teoria, modelli interpretativi specifici.

\section{Riflessioni conclusive: le opportunità dell'attivismo e il distacco dai partiti}

Le organizzazioni giovanili dei partiti - vuoi perché soffocate dalle gerarchie interne, vuoi per propria inerzia - si segnalano molto raramente per iniziative che tocchino le corde più sensibili degli interessi e delle passioni dei giovani. Ad uno sguardo retrospettivo, queste organizzazioni si sono ritagliate piuttosto uno spazio nel sistema politico italiano quali bacini di reclutamento di politici di carriera.

$\grave{E}$ noto che dal secondo dopoguerra ai primi anni novanta la classe politica nazionale - a tutti i livelli - è stata costituita in larga parte da individui che hanno percorso una carriera cominciata in giovane età in strutture di partito (sul personale ministeriale, Calise e Mannheimer 1982; sui parlamentari, Cotta 1979, 171; Mastropaolo 1994, 463; sulla classe politica municipale, Bettin e Magnier 1991, 123 e ss.; Bettin e Magnier 1995, 108-111)26. Più nel dettaglio, vale la pena sottolineare che le organizzazioni partitiche e parapartitiche giovanili hanno rappresentato la porta d'accesso alla carriera politica più comune persino per i parlamentari eletti nel 1992 e nel 1994 - cioè, nelle consultazioni elettorali in cui il vento dell'anti-party politics è spirato forte come mai prima nel Paese. Quasi il 30\% dei deputati e senatori in carica tra il 1992 e il 1996 e il 50\% dell'élite politica vera e propria - ossia, dei membri del parlamento che ricoprono o hanno ricoperto un incarico ai massimi vertici delle istituzioni: ex presidenti della repubblica, del consiglio o di un ramo del parlamento, segretari di partito o ministri - ha svolto il proprio apprendistato politico negli organismi giovanili dei partiti (Recchi 1997b, 370 e

26 Le analisi citate sono precedenti il biennio 1993-94, caratterizzato da un forte turnover dei rappresentanti politici a tutti $\mathrm{i}$ livelli. E tuttavia già nelle elezioni politiche del 1996 le opportunità di ingresso ed ascesa in politica per outsider e dilettanti risultano di nuovo molto diminuite, tanto da far concludere che «all'interno della classe politica quel po' di novità [...] che si era affacciato nel 1994 è stato in gran parte riassorbito» (Mastropaolo 1996, 505; cfr. anche Verzichelli 1996 e Nevola 1997). In una prospettiva più generale, probabilmente, i processi di circolazione delle élites politiche di breve periodo non interrompono la tendenza a lungo termine alla professionalizzazione della rappresentanza politica preconizzata originariamente da Schumpeter (1964, 272; nel caso italiano l'andamento di questa tendenza è mostrato da Cotta et al. 1998). 
377). Peraltro, il ruolo delle organizzazioni giovanili di partito quali strutture votate al reclutamento iniziale dei politici di professione risalta anche all'analisi delle carriere successive dei loro ex dirigenti. Oltre il $75 \%$ di coloro che, fra il 1974 e il 1977, furono membri delle direzioni nazionali o dei comitati centrali (nonché più del 60\% degli ex membri dei consigli nazionali) delle federazioni giovanili del Pci, del Psi e della Dc hanno raggiunto nei primi anni novanta una posizione di rilievo politico-amministrativo tale da essere inclusi in almeno uno dei principali who's who italiani (Recchi 1997a, 90-96).

Questi precedenti, probabilmente, hanno importanti ripercussioni sulla percezione della militanza partitica da parte della popolazione giovanile. Se essa si configura come un'attività dagli esiti professionalizzanti all'interno di un ambiente organizzativo che, in molti casi, tende all'esclusività dell'impegno, il suo potere attrattivo ne risulta inevitabilmente limitato. I dati sulle carriere e i risultati dell'analisi empirica sui giovani attivisti si saldano a corroborare questa interpretazione. Non è un caso che il modello che si è dimostrato più adatto a spiegare le differenze tra $\mathrm{i}$ giovani attivisti di partito e $\mathrm{i}$ loro coetanei descriva più un pattern di scelta di carriera che una dinamica di mobilitazione politico-ideologica. Proprio perché tradizionalmente ha costituito un buon trampolino per l'avvio di una carriera politi$\mathrm{ca}, \mathrm{il}$ coinvolgimento in un partito finisce per attrarre solamente chi è dotato di risorse che possono risultare utili in una chiave di professionalizzazione di tale coinvolgimento. Siccome queste risorse hanno una forte specificità (si pensi alla politicizzazione familiare, al legame con un potenziale sponsor politico, alla disponibilità di un ampio volume di capitale sociale), coloro che ne sono sprovvisti - cioè, quasi tutti - sono fortemente incentivati a scartare senza indugi l'ipotesi di partecipare in prima persona alla vita dei partiti. Natura e prospettive dell'attivismo di partito, in definitiva, sembrano scoraggiare in anticipo, con modalità sottilmente discriminatorie ${ }^{27}$, il possibile impegno politico

27 Per quanto osservato nel corso della ricerca sul campo, sembra di dover escludere che le organizzazioni giovanili di partito esaminate esercitino un filtro selettivo su chi è intenzionato a farne parte. Purtuttavia, si ritiene che l'autoselezione per l'attivazione politica in tali organizzazioni non sia esente da una sorta di discriminazione preventiva derivante dalla «formazione adattiva di preferenze» che aggiustano le aspirazioni soggettive alle possibilità oggettive (Elster 1989, 133 e ss.; cfr. anche Bourdieu 1977, 77). Nel nostro caso, si può presumere che eventuali aspirazioni all'impegno politico vengano accantonate per effetto dell'indisponibilità delle risorse che definiscono il modello di attivazione prevalente. 
dei giovani che non possono permettersi o semplicemente non vogliono accettare di giocare il ruolo dell'attivista così come è venuto a configurarsi nei partiti italiani. Finisce così per consolidarsi ulteriormente l'immagine dei partiti quali entità remote $\mathrm{e}$ non gratae nell'orizzonte politico del mondo giovanile.

\section{Riferimenti bibliografici}

Aldrich, J.H. (1997), Positive Theory and Voice and Equality, in «American Political Science Review», n. 3, pp. 421-423.

Barber, J.D. (1965), The Lawmakers, New Haven, Yale University Press.

Barton, A.H. e R.W. Parsons (1977), Measuring Belief System Structure, in «Public Opinion Quarterly», n. 2, pp. 159-180.

Begg, C.B. e R. Gray (1984), Calculation of Polychotomous Logistic Regression Parameters Using Individualized Regressions, in «Biometrika», n. 1, pp. 11-18.

Bertaux, D. (1995), Social Genealogies Commented On and Compared: An Instrument for Observing Social Mobility Processes in the Longue Durée, in «Current Sociology», n. 2-3, pp. 69-88.

Bettin, G. e A. Magnier (1991), Chi governa la città?, Padova, Cedam.

- (1995), I nuovi sindaci: come cambia una carriera politica, in «Rivista Italiana di Scienza Politica», n. 1, pp. 91-118.

Bourdieu, P. (1977), Outline of a Theory of Practice, Cambridge, Cambridge University Press.

- (1986), The Forms of Capital, in J.G. Richardson (a cura di), Handbook of Theory and Research for the Sociology of Education, New York, Greenwood.

Brady, H.E., S. Verba e K. Lehman Schlozman (1995), Beyond SES: A Resource Model of Political Participation, in «American Political Science Review», n. 2, pp. 271-294.

Browning, R.P. e H. Jacob (1964), Power Motivation and the Political Personality, in «Public Opinion Quarterly», n. 1, pp. 75-90.

Buzzi, C., A. Cavalli e A. de Lillo (1997), Giovani verso il Duemila. Quarto rapporto Iard sulla condizione giovanile in Italia, Bologna, Il Mulino.

Calise, M. e R. Mannheimer (1982), Governanti in Italia, Bologna, Il Mulino.

Carlson, J.M. e M.S. Hyde (1980), Personality and Political Recruitment: Actualization or Compensation?, in «Journal of Psychology», n. 1, pp. 117-120.

Cavalli, A. e A. de Lillo (a cura di) (1993), Giovani anni 90. Terzo rapporto Iard sulla condizione giovanile in Italia, Bologna, Il Mulino.

Clarke, J.W. e M.M. Donovan (1980), Personal Needs and Political In- 
centives: Some Observations on Self-Esteem, in «American Journal of Political Science», n. 3, pp. 536-552.

Clarke, H.D., A. Kornberg e J. Lee (1975), Ontario Student Party Activists: A Note on Differential Participation in a Voluntary Organization, in «Canadian Review of Sociology and Anthropology», $\mathrm{n}$. 2, pp. 213-220.

Cobalti, A. e A. Schizzerotto (1994), La mobilità sociale in Italia, Bologna, Il Mulino.

Cotta, M. (1979), Classe politica e parlamento in Italia 1946-1976, Bologna, Il Mulino.

Cotta, M., A. Mastropaolo e L. Verzichelli (1998), Italy: Parliamentary Elite Transformations along the Discontinuous Road of Democratization, in M. Cotta e H. Best (a cura di), The European Legislature. Long Term Transformations of Parliamentary Elites, Oxford, Oxford University Press (in corso di pubblicazione).

Darcy, R., S. Welch e J. Clark (1987), Women, Elections, and Representation, New York, Longman.

Dowse, R.E. e J.A. Hughes (1977), Pre-Adult Origins of Adult Political Activity: A Sour Note, in C. Crouch (a cura di), Participation in Politics. British Political Sociology Yearbook 3, London, Croom Helm.

Elster, J. (1989 [1983]), Uva acerba. Versioni non ortodosse della razionalità, Milano, Feltrinelli.

Erikson, R. e J.H. Goldthorpe (1992), The Constant Flux: A Study of Class Mobility in Industrial Societies, Oxford, Clarendon Press.

Greenstein, F. (1977), Introduction, in Lasswell.

Guadagnini, M. (1993), A 'Partitocrazia' Without Women: the Case of the Italian Party System, in J. Lovenduski e P. Norris (a cura di), Gender and Party Politics, London, Sage.

Hudon, R. e B.-P. Hébert (1994), Porquoi les jeunes s'intéressaient-ils à la politique, in R. Hudon e B. Fournier (a cura di), Jeunesse et politique. Conceptions de la politique en Amérique du Nord et en Europe, vol. I, Sainte-Foy-Paris, Presses de l'Université LavalL'Harmattan.

Iremonger, L. (1970), The Fiery Chariot: British Prime Ministers and the Search for Love, London, Secker \& Warburg.

Istat (1995), Popolazione e abitazioni. $13^{\circ}$ censimento generale della popolazione. Fascicolo nazionale Italia 1991, Roma, Istat.

- (1996), Cultura socialità tempo libero. Indagine multiscopo sulle famiglie. Anni 1993-1994, Roma, Istat.

Jaccard, J., R. Turrisi e C.K. Wan (1990), Interaction Effects in Multiple Regression, Newbury Park, Sage.

Janoski, T. e J. Wilson (1995), Pathways to Voluntarism: Family Socialization and Status Transmission Models, in «Social Forces», n. 1, pp. 271-292.

Kearney, R.N. (1984), The Mentor in the Commencement of a Political 
Career: The case of Subbas Chandra Bose and C.R. Das, in «Journal of Political and Military Sociology», n. 1, pp. 37-47.

Kellerman, B. (1978), Mentoring in Political Life: The Case of Willy Brandt, in «American Political Science Review», n. 2, pp. 422-433.

Kendall, M. (1980), Multivariate Analysis, London, Griffin \& Co.

Knutson, J.N. (1973), Personality in the Study of Politics, in Id. (a cura di), Handbook of Political Psychology, San Francisco, Jossey-Bass.

Lane, R.E. (1959), Political Life: Why People Get Involved in Politics, New York, The Free Press.

Lasswell, H.D. (1977 [1930]), Psychopathology and Politics, Chicago, University of Chicago Press.

- (1948), Power and Personality, New York, Norton.

- (1950 [1936]), Politics: Who Gets What, When, and How, New York, Peter Smith.

Levinson, D.J. (1978), The Seasons of a Man's Life, New York, Ballantine.

Marsh, D. (1971), Political Socialization: The Implicit Assumptions Questioned, in «British Journal of Political Science», n. 4, pp. 453-465.

Marvick, D. e C.R. Nixon (1961), Recruitment Contrasts in Rival Campaign Groups, in D. Marvick (a cura di), Political DecisionMakers: Recruitment and Performance, New York, The Free Press.

Mastropaolo, A. (1994), Le elezioni politiche del marzo 1994. Vecchio e nuovo nel Parlamento italiano, in «Italia Contemporanea», n. 169, pp. 461-470.

- (1996), La classe politica parlamentare tra rivoluzione e restaurazio$n e$, in «Italia Contemporanea», n. 204, pp. 497-508.

Merelman, R.M. e G. King (1986), The Development of Political Activists: Toward a Model of Early Learning, in «Social Science Quarterly», n. 3, pp. 473-490.

Milbrath, L.W. e M.L. Goel (1977), Political Participation, Lanham, University Press of America.

Nevola, G. (1997), Alla ricerca di un ceto politico. I candidati del ciclo 1994-1996 tra innovazione e consolidamento, in P. Corbetta e A.M.L. Parisi (a cura di), Cavalieri e fanti. Proposte e proponenti nelle elezioni del 1994 e del 1996, Bologna, Il Mulino.

Newton, K. (1997), Social Capital and Democracy, in «American Behavioral Scientist», n. 5, pp. 575-586.

Pizzorno, A. (1993 [1966]), Condizioni della partecipazione politica, in Id., Le radici della politica assoluta e altri saggi, Milano, Feltrinelli.

Prentice, R.L. e R. Pyke (1979), Logistic Disease Incidence Models and Case-Control Studies, in «Biometrika», n. 3, pp. 403-411.

Press, S.J. e S. Wilson (1978), Choosing Between Logistic Regression and Discriminant Analysis, in «Journal of the American Statistical Association», n. 364, pp. 699-705.

Prewitt, K. (1965), Political Socialization and Leadership Selection, in 
«Annals of the American Academy of Political and Social Sciences», n. 361, pp. 96-111.

Putnam, R.D. (1976), The Comparative Study of Political Elites, Englewood Cliffs, Prentice-Hall.

Recchi, E. (1997a), Giovani politici, Padova, Cedam.

- (1997b), La formazione della classe politica parlamentare (19921996) e il «regime dei partiti»: permanenza o cambiamento?, in G. Bettin (a cura di), Politica e società. Studi in onore di Luciano Cavalli, Padova, Cedam.

Renshon, S.A. (1989), Psychological Perspectives on Theories of Adult Development and the Political Socialization of Leaders, in R.S. Sigel (a cura di), Political Learning in Adulthood: A Sourcebook of Theory and Research, Chicago, University of Chicago Press.

Rotter, J.B. (1966), Generalized Expectancies for Internal vs. External Control of Reinforcement, in «Psychological Monographs», n. 1, pp. 1-28.

Salisbury, R. (1965), The Urban Party Organization Member, in «Public Opinion Quarterly», n. 4, pp. 553-565.

Schumpeter, J. (1964 [1942]), Capitalismo socialismo democrazia, Milano, Comunità.

Searing, D., J.J. Schwartz e A.E. Lind (1973), The Structuring Principle: Political Socialization and Belief Systems, in «American Political Science Review», n. 2, pp. 415-432.

Searing, D., G. Wright e G. Rabinowitz (1976), The Primacy Principle: Attitude Change and Political Socialization, in «British Journal of Political Science», n. 1, pp. 83-113.

Stone, W.F. (1974), The Psychology of Politics, New York, The Free Press.

Stryker, S. (1981), Symbolic Interactionism: Themes and Variations, in R.E. Turner e M. Rosenberg (a cura di), Social Psychology: Sociological Perspectives, New York, Basic Books.

Tapper, T. (1976), Political Education and Stability. Elite Responses to Political Conflict, London, Wiley.

Tetlock, P.E. (1985), Integrative Complexity of Policy Reasoning, in S. Kraus e R.M. Perloff (a cura di), Mass Media and Political Thought, Beverly Hills, Sage.

Tullio-Altan, C. e A. Marradi (1976), Valori, classi sociali, scelte politiche. Indagine sulla gioventù degli anni settanta, Milano, Bompiani.

Turi, P. (1997), I giovani e $i$ partiti, paper presentato al seminario «Valori politici e nuove generazioni nell'Europa contemporanea», Centro Interuniversitario di Sociologia Politica, Firenze, 25-26 settembre.

Verba, S., N.H. Nie e J. Kim (1978), Participation and Political Equality: A Seven Nation Comparison, Cambridge, Cambridge University Press.

Verba, S., K.L. Schlozman e H.E. Brady (1997), Solving the Puzzle of 
Rational Participation: Lessons from the Citizen Participation Stu$d y$, paper presentato all'Annual Meeting dell'American Political Science Association, Washington, 28-31 agosto.

Verzichelli, L. (1996), La classe politica della transizione, in «Rivista Italiana di Scienza Politica», n. 3, pp. 727-768.

Xie, Y. e C.F. Manski (1989), The Logit Model and Response-Based Samples, in «Sociological Methods \& Research», n. 3, pp. 283 302.

Ziller, R.C., W.F. Stone, R.M. Jackson e N.J. Terbovic (1977), SelfOther Orientations and Political Behavior, in M.G. Hermann (a cura di), A Psychological Examination of Political Leaders, New York, The Free Press. 